\title{
Selective Intraoperative Transcystic Cholangiogaraphy in Laparoscopic Cholecystectomy, 3 Years' Experience Ayman Helmy Ibrahim ${ }^{1 *}$, Mohamed A. Abdellatif ${ }^{2}$ \\ Departments of ${ }^{1}$ General Surgery and ${ }^{2}$ Diagnostic Radiology and Medical Imaging, Faculty of Medicine, Al-Azhar University, Cairo, Egypt \\ *Corresponding author: Ayman Helmy Ibrahim, email: aymanmohamedmed.b@azhar.edu.eg
}

\begin{abstract}
Background: Use of intraoperative cholangiography (IOC), a contrast-based examination of the biliary system during laparoscopic cholecystectomy (LC) still represents a valuable approach to illustrate biliary duct anatomy, diagnose obstruction and avoid complications.

Objective: The aim of work was to detect the advantages of IOC when used in selected cases during LC in diagnosis of biliary obstruction and prevention of biliary injuries.

Patients and methods: 29 patients with specific criteria were included in the study to perform LC and IOC after preoperative magnetic resonance cholangio pancreatogaraphy (MRCP). Follow-up was done by clinical examination, serum bilirubin and U/S for one year. Collected data had been statistically analyzed.

Results: IOC resulted in significant increase in mean surgical time with longer time in cases with pericholecystic fluid and mucocele. IOC still has a higher specificity, sensitivity and positive predictive value (PPV) (100, $87.5 \& 100 \%$ respectively) over MRCP $(65,77.8 \& 50 \%$ respectively) in prediction of choledocholithiasis. U/S, MRCP \& IOC had high negative predictive values (NPV), good negative tests. History of obstructive jaundice had a low sensitivity, specificity and PPV $(71.5,63.6 \& 38.5)$ but had a high NPV (87.5\%) in detection of choledocholithiasis. Normal IOC saved 8 patients with history of obstructive jaundice from unnecessary endoscopic retrograde cholangiopancreatography (ERCP). No biliary injuries had been reported.

Conclusion: selective IOC in high risk patients is useful in detection of choledocholithiasis, avoidance of ERCP in patients with non-specific common bile duct (CBD) dilatation and prevention of biliary injuries in cases with high risk for injury.
\end{abstract}

Keywords: Laparoscopic cholecystectomy, MRCP, selective intraoperative cholangiogram, biliary injuries.

\section{INTRODUCTION}

Gall stone disease is one of the most prevalent clinical problems all around the world. The incidence of gallstones is three folds more common in females than in males ${ }^{(1-3)} .12 \%$ of patients with symptomatic gall stones have stones in the common bile duct (CBD) that may be asymptomatic, but it may cause serious complications as obstructive jaundice, cholangitis and pancreatitis. Investigations should be done to rule out choledocholithiasis prior to surgery to diagnose choledocholithiasis if present and avoid future complications of missed stones ${ }^{(4)}$. Specificity and sensitivity and of laboratory tests are low, ultrasonography (U/S) is a durable, low cost, noninvasive bed-side test, with no use of ionizing radiation and can measure the diameters bile ducts, however, approximately $65 \%$ of bile duct calculi are not detected by U/S due to interference with bowel gases and also because of being a highly operator dependent procedure (3-5). Magnetic resonance cholangiopancreatography (MRCP) also has an important well-established role in identifying uncertain anatomy and visualizing the bile duct stones ${ }^{(6,7)}$. IOC is the gold standard procedure in the diagnosis of choledocholithiasis as it maps the anatomy of biliary tree and detect pathological conditions ${ }^{(8,9)}$. Being an invasive and contrast-based procedure, routine versus selective use of IOC is a matter of controversy in many literature as it may increase intra and post-operative complications.
Studies were held to use a safer, more cost effective, less invasive procedures with less

complication which have a high sensitivity and specificity in prediction of choledocholithiasis to replace IOC ${ }^{(9-11)}$. This study present an experience on suspicious patients for choledocholithiasis and/or difficult surgery with use of preoperative MRCP followed by selective use of IOC to detect additional benefits from use and reliability of different risk factors for prediction choledocholithiasis and also to try to answer the question: Can MRCP eliminate the use of IOC?

The aim of this study was to evaluate the protective effect of selective use of IOC during laparoscopic cholecystectomy (LC).

\section{PATIENTS AND METHODS}

This study included a total of twenty-nine patients with symptomatic gall bladder stones and suspicion of CBD stones, attending at General Surgery Department of Al-Azhar University Hospitals; Al-Hussein and Bab El-Sharia Hospitals, Cairo, Egypt. This study was conducted between October 2015 to September 2018.

\section{Ethical approval:}

Approval of the ethical committee of the university hospitals was obtained. Written informed consent from all the subjects were obtained. 
After taking history and clinical examination, routine laboratory chemical tests including liver enzymes and serum bilirubin were assessed for the patients. Then abdominal U/S was performed to diagnose the presence of gallstones and detect CBD stone or bile duct dilation and/or anomalies.

Inclusion criteria included: history of obstructive jaundice, elevated serum direct and/or total bilirubin or alkaline phosphatase, dilated common bile duct (CBD) preoperatively, inexistent or short cystic duct, obscured operative anatomy due to marked adhesions or anomalies of biliary ducts.

Exclusion criteria included the presence of obstructive jaundice or acute cholecystitis and contraindications of cholangiography as idiosyncrasy to the contrast.

The patients with high suspicion to choledocholithiasis according to previous criteria received a preoperative MRCP (fig. 1) which were interpreted by single radiologist then scheduled for laparoscopic cholecystectomy and undergone Intraoperative cholangiography (IOC) with subsequent cholecystectomy.

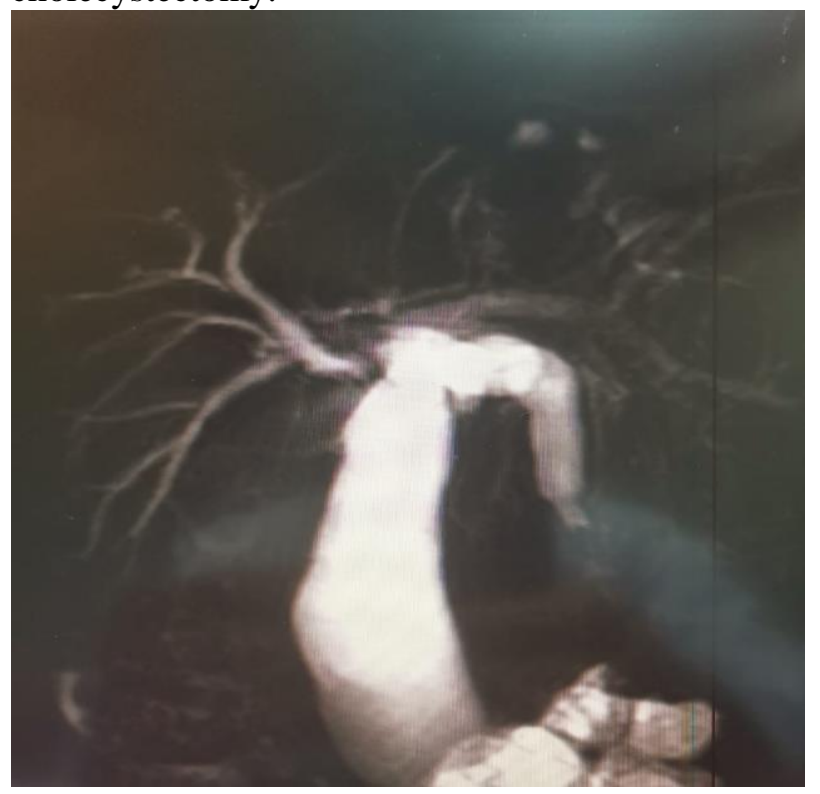

Fig. (1): preoperative cholangiogram showing dilated CBD, distal end CBD filling defect (stone) and minimal intrahepatic biliary dilation.

The intraoperative cholangiogram was done through a small incision in the cystic duct (Fig. 2). Cannulation of the cystic duct using silicone catheter was performed, diluted contrast material was injected, and films were taken (Fig. 3). IOC films were examined and interpreted by the same radiologist who was not informed by MRCP scan and chemical test results of the examined case (Fig. 4 \& 5). Surgeries were performed by one surgeon; IOC was initially done, and the film was reported and analyzed by the blinded radiologist.

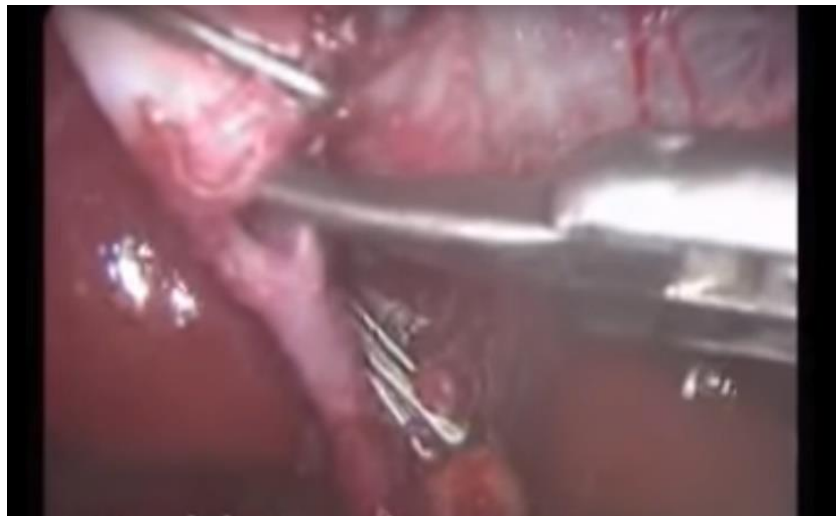

Fig. (2): Incision of cystic duct

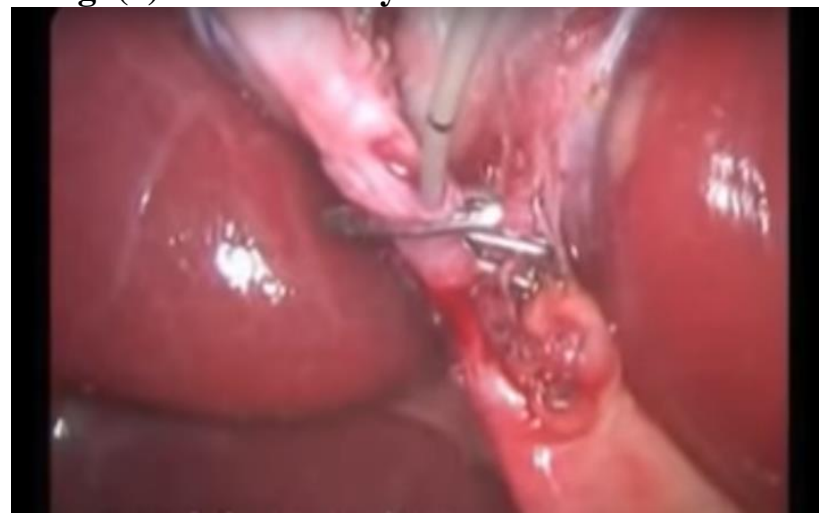

Fig. (3): Cannulation of cystic duct

Follow-up was done clinically and by serum bilirubin (total \& direct) with abdominal U/S for one year. All data were collected and analyzed as regard perioperative details and clinical outcomes.
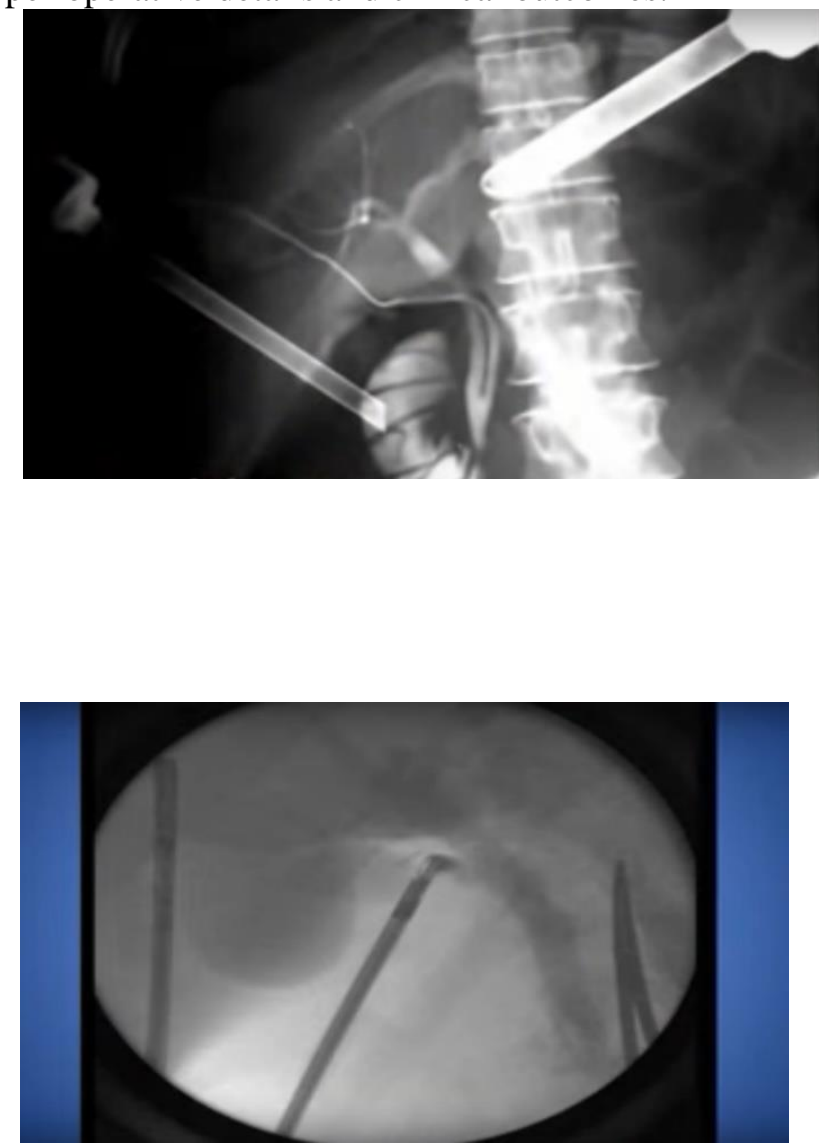

Fig. (4) \& (5): images of intraoperative cholangiogram showing dilated biliary tree. 


\section{Statistical Analysis}

Data were expressed in the forms of mean standard deviation (SD), sensitivity and specificity, positive and negative predictive values that were calculated for different parameters. Sensitivity was defined as the ability of the test to detect patients with CBD stones from the total number of patients with confirmed CBD stones. Specificity was defined as the ability of the test to detect patients without CBD from the total number of patients without IOC-proven CBD stones. Positive and negative predictive values of the test were determined by the proportion of the test positive or negative results confirmed by IOC or postoperative follow-up. A significant difference was considered when $p$-value $<0.05$. Statistical analyses of collected data were performed by the SPSS software computer program (SPSS17, SPSS Inc. Chicago, IL).

\section{RESULTS}

The study included 29 patients; 19 females and 10 males. Patient ages ranged from 29-62 with a mean age of $45 \pm 9$ SD years. The mean hospital stay was $1.5 \pm 0.9$ days. No significant differences were detected as regard patient age, gender or hospital stay. The time of the whole procedure (LC+IOC) ranged from 51 to $195 \mathrm{~min}$ and the mean of $115.8 \pm 26.3 \mathrm{~min}$. The mean time of IOC ranged from 20 to $42 \mathrm{~min}$ and the mean of $29.5 \pm 9.6 \mathrm{~min}$. IOC resulted in significant increase in mean surgical time $(P=0.025)$. Time showed longer values in cases with positive $\mathrm{C}$-reactive protein, pericholecystic fluid, marked adhesions and mucocele with statistically significant differences (Table 1).

Table (1): time of the whole procedure and IOC.

\begin{tabular}{|l|l|l|l|}
\hline Time & LP+ IOC & IOC & $P$ value \\
\hline $\begin{array}{l}\text { Range } \\
\text { (min) }\end{array}$ & $51-195$ & $20-42$ & $\begin{array}{l}P= \\
0.025\end{array}$ \\
\cline { 1 - 3 } Mean + SD & $115.8 \pm 26.3$ & $29.5 \pm 9.6$ & \\
\hline
\end{tabular}

Preoperatively, 19 patients (65.5\%) had dilated CBD and $10(34.5 \%)$ cases had normal caliber of CBD by U/S examination. Preoperative MRPC was positive (dilated biliary tract with or without filing defects) in 14 cases $(48.3 \%)$ and 15 cases $(51.7 \%)$ had normal MRCP findings (Fig. 6). Abnormal cholangiograms in the study were noticed and further interference was needed only in 7 cases $(24.1 \%) ; 5$ cases showed multiple filling defects due to CBD stones and 2 cases showed distal tapering due to benign stricture. These cases needed further interventions included endoscopic retrograde cholangiopancreatography (ERCP), which was done intraoperatively in 2 cases and postoperatively in 3 cases, and CBD exploration which was done by open technique in the remaining 2 cases. Among the 15 cases $(51.7 \%)$ that showed preoperative normal MRCP, one case had abnormal cholangiogram and treated by postoperative ERCP 24 hours after surgery. During the postoperative follow-up, single case developed obstructive jaundice one month after operation despite normal U/S, MRCP and IOC; and treated by ERCP stone extraction (Table 2).

Sensitivity of U/S, MRCP and IOC were $87.5 \%, 77.8 \%$ and $87.5 \%$ respectively. Specificity of these methods revealed $42.8 \%$ for U/S, $65 \%$ for MRCP and $100 \%$ for IOC (Fig. 7). Positive predictive values (PPV) of U/S, MRCP and IOC were $36.8 \%, 50 \%$ and $100 \%$ while negative predictive values (NPV) were 90\% for U/S, $86.7 \%$ for MRCP and $95.5 \%$ for IOC (Fig. 8). There was significant differences between results of preoperative investigations (including U/S and MRCP) and IOC in detection of choledocholithiasis.

Table (2): Results of U/S, MRCP and IOC tests in prediction of choledochlithiasis.

\begin{tabular}{|l|l|l|l|l|}
\hline Test & U/S & MRCP & IOC & P value \\
\hline Positive test & 19 & 14 & 7 & $P=$ \\
\hline $\begin{array}{l}\text { Negative } \\
\text { test }\end{array}$ & 10 & 15 & 22 & 0.035 \\
\hline TP & 7 & 7 & 7 & \\
\hline TN & 9 & 13 & 21 \\
\hline FP & 12 & 7 & 0 & \\
\hline FN & 1 & 2 & 1 \\
\hline Sensetivity & $\begin{array}{l}77.5 \\
\%\end{array}$ & $77.5 \%$ & $87.5 \%$ & \\
\hline Specificity & $\begin{array}{l}4.8 \\
\%\end{array}$ & $65 \%$ & $100 \%$ & \\
\hline PPV & $\begin{array}{l}6.8 \\
\%\end{array}$ & $50 \%$ & $100 \%$ & \\
\hline NPV & $90 \%$ & $86.7 \%$ & $95.5 \%$ & \\
\hline
\end{tabular}

Positive test $=$ dilated $\mathrm{CBD}$, negative test $=$ normal CBD, $T P=$ true positive cases, $T N$ $=$ true negative cases, $\mathrm{FP}=$ false positive cases, $\mathrm{FN}=$ false negative cases, $\mathrm{PPV}=$ positive predictive value, $\mathrm{NPV}=$ negative predictive value. 


\section{Results of U/S, MRCP, IOC}

25

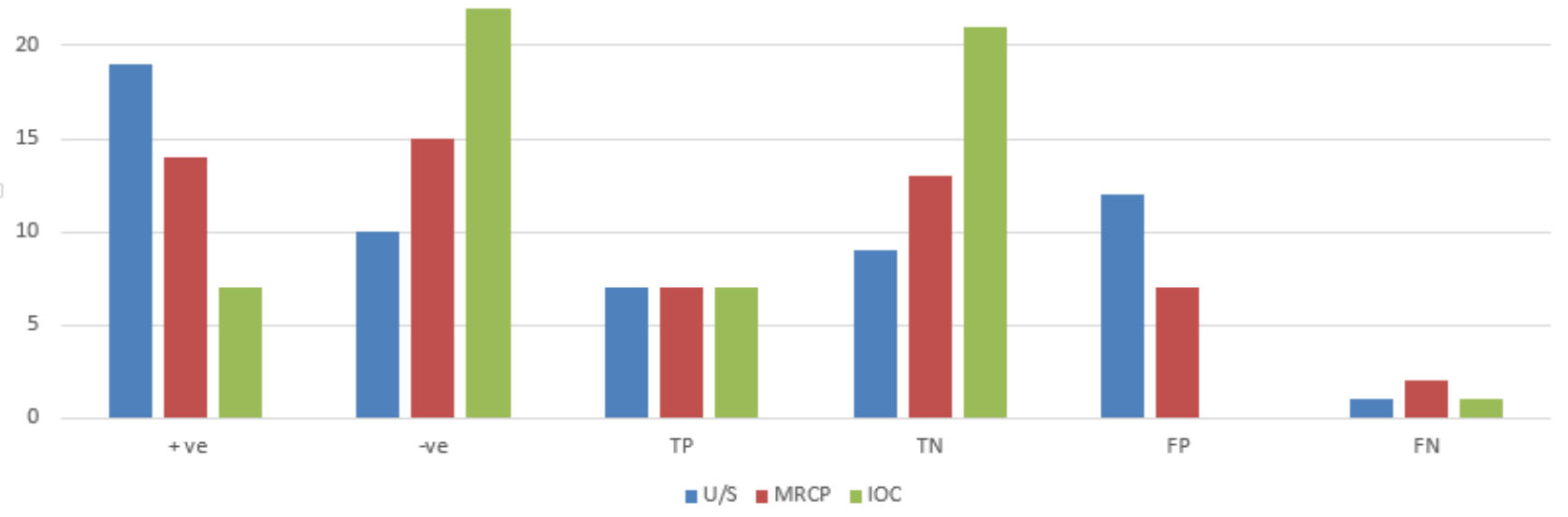

Fig (6): results of U/S, MRCP, IOC in the study

\section{POSITIVE AND NEGATIVE PREDICTIVE VALUES OF \\ U/S - MRCP - IOC}

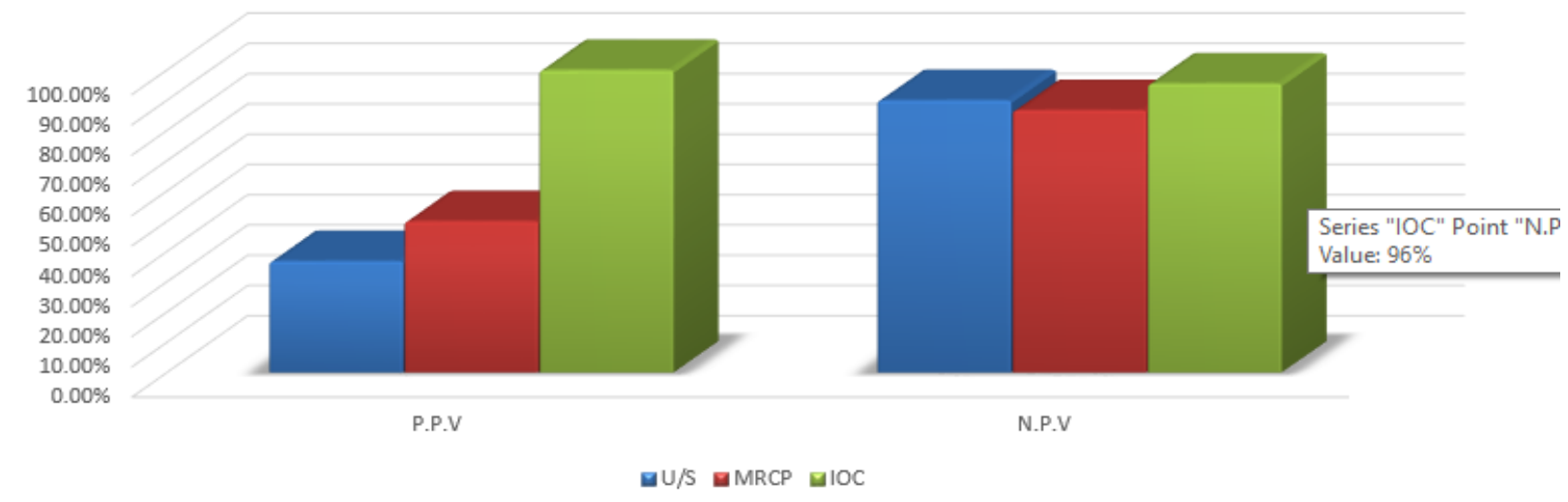

Fig (7): PPV and NPV of U/S, MRCP, IOC in the study

\section{SENSETIVITY AND SPECIFICITY OF \\ U/S - MRCP - IOC}

$120.00 \%$

$100.00 \%$

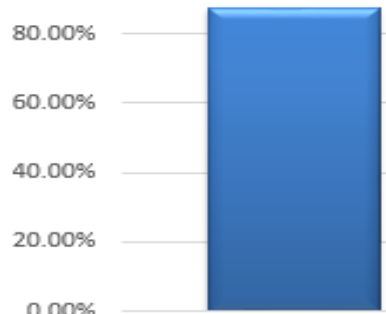

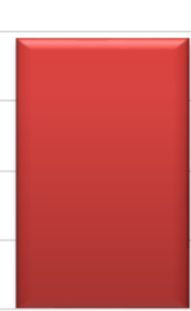

Sensetivity

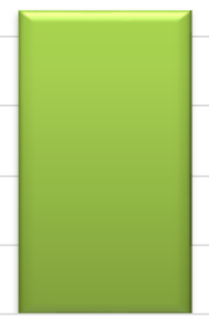

$\square \mathrm{U} / \mathrm{S} \square \mathrm{MRCP} \square \mathrm{IOC}$

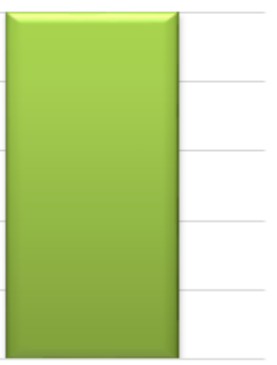

sepecivity

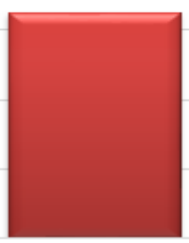

Fig (8): sensitivity and specificity of U/S, MRCP, IOC in the study 
History of obstructive jaundice was reported in 13 (44.9\%) of the cases while 16 cases $(55.1 \%)$ did not report past history of jaundice. Two cases showed abnormal IOC due to stones in CBD despite no history of obstructive jaundice. Among 13 patients with history of obstructive jaundice, 8 patients showed normal IOC. In this experience, history of obstructive jaundice had a low sensitivity (71.5\%) and specificity (63.6\%) and also low positive predictive value (38.5\%) in detection of choledocholithiasis with a high (87.5) negative predictive value (Table 3).

Table (3): Results of + ve history of obstructive jaundice in prediction of choledochlithiasis.

\begin{tabular}{|l|l|}
\hline Results & $\begin{array}{c}\text { History of } \\
\text { obstructive } \\
\text { jaundice }\end{array}$ \\
\hline Positive cases & 13 \\
\hline Negative cases & 16 \\
\hline TP & 5 \\
\hline TN & 14 \\
\hline FP & 8 \\
\hline FN & 2 \\
\hline Sensetivity & $71.5 \%$ \\
\hline Specificity & $63.6 \%$ \\
\hline PPV & $38.5 \%$ \\
\hline NPV & $87.5 \%$ \\
\hline
\end{tabular}

\section{Positive case $\boldsymbol{=}$ history of obstructive jaundice, negative test $=$ no history of obstructive jaundice, $\mathrm{TP}=$ true positive cases, $\mathrm{TN}=$ true negative cases, $\mathrm{FP}=$ false positive cases, $\mathrm{FN}=$ false negative cases, $\mathrm{PPV}=$ positive predictive value, $\mathrm{NPV}=$ negative predictive value.}

At time of surgery, 8 cases $(27.5 \%)$ showed high total bilirubin, 12 cases $(41.3 \%)$ had high direct bilirubin; 3 cases $(10.3 \%)$ had mucocele and $4(13.7 \%)$ cases had pericholecystic fluid. No cases of biliary duct injuries were neither reported intraoperative nor detected in the postoperative follow up in this study.

\section{DISCUSSION}

Use of IOC has been a matter of debate in previous literature and studies. Many surgeons routinely use IOC in cases of $\mathrm{LC}$ while others prefer to perform IOC selectively in patients with certain criteria detected preoperative or intraoperative. This debate was obvious in laparoscopic surgical guidelines, The SAGES guidelines of clinical applications of laparoscopic biliary surgery recommend that IOC has proved to decrease the risk of bile injuries when routinely used and to allow access for therapeutic intervention into the biliary tree ${ }^{(12)}$. On other hand, recent guidelines from European Association of Endoscopic Surgery (EAES) states that the available literature could not recommend routine use of IOC which remains controversial. The EAES guideline, however, indicated that IOC has important role that allows for early detection of bile duct injuries (BDIs) if correctly interpreted ${ }^{(13)}$.

Study of Amott et al. adopted the routine use of IOC in LC as they reported that in patients of the routine IOC group, 94 underwent IOC but CBD stones were detected in 12 of them while in the selective group, IOC was done in 34 of 45 cases and CBD stones were shown in 5 cases with no significant differences between both policies of IOC in the mean operative time, CBD stone detection rates or biliary injury so, policy of routine IOC has been adopted ${ }^{(14)}$. In the metaanalysis of Ludwig et al., they analyzed forty case series included 327523 cases and reported that routine use of IOC is not only associated with a lower incidence of bile duct injuries ( 0.21 vs. $0.43 \%)$ but also with higher rates of initial detection at the time of surgery $(87 \text { vs. } 44.5 \%)^{(15)}$. Flum et al., reported that incidence of BDIs decreased from 3.2 to 1.7 per 1000 operations with routine IOC and identified a statistical significance of 1.7-fold increase in the risk of BDIs if IOC was not routinely used ${ }^{(16)}$

Other studies did not recommend routine use of IOC in all cases of LC. There were no significant differences between routine and selective groups as regard BDIs, mortality rates and CBD stone detection while the rate of overall complications was higher in routine IOC group. IOC has many disadvantages including prolongation of operation and anesthesia time, significant added costs and more peri-surgical procedures. Use of ionizing radiation and contrast together with surgical dissection and incision of biliary tree should be considered as risk factors for more hazardous effects that increase incidence of intra and postoperative complications ${ }^{(17,18)}$. Many surgeons do not prefer routine IOC as it is associated and with increased overall complications. These data suggest that routine IOC may not improve outcomes ${ }^{(19)}$.

Sheffield et al. study in 2013 to detect the association of IOC use and BDIs incidence determined that there had been no association between use of IOC and incidence of BDIs. The Ford et al. study did not identify any added benefits of IOC use for the prevention of BDIs. These studies recommended selection of cases susceptible to BDIs for IOC instead of routine use ${ }^{(17,18)}$.

On other hand, the presence of CBD stones (choledocholithiasis) in patients with symptomatic gall stones is up to $12 \%$ that may be asymptomatic in most cases leading to later serious complications so, CBD stones should be detected using different clinical, laboratory and radiological methods and treated (4). Many studies identified the higher sensitivity and positive predictive value of Magnetic resonance cholangiopancreatography (MRCP) than U/S and other tests for preoperative detection of choledocholithiasis (3-7). 
In the current study, selective IOC was done in high susceptible patients for choledocholithiasis or BDIs instead of routine use as it is an invasive procedure that needs anesthesia, prolongs the duration of surgery and anesthesia, entails surgical dissection, incision of bile ducts, cannulation using catheters, injection of contrast and use of ionizing radiation so, it may increase intra and post-operative complications. The mean time of the whole procedure $(\mathrm{LC}+\mathrm{IOC})$ ranged from 51 to $195 \mathrm{~min}$ and the mean of $115.8 \pm 26.3$. The mean time of IOC ranged from 20 to $42 \mathrm{~min}$ and the mean of $29.5 \pm 9.6$. IOC resulted in significant increase in mean surgical time. The time showed longer values in cases with positive $\mathrm{C}$-reactive protein, pericholecystic fluid, marked adhesions and mucocele with statistically significant differences.

Preoperative MRCP was performed for all cases to assess its role in prediction of choledocholithiasis as it has advantages of being a noninvasive, non-contrast dependent procedure which gives a high resolution mapping of bile ducts without use of any ionizing radiation that helps in identifying uncertain anatomy and visualizing the bile duct stones $(3,7,8)$.

Sensitivity, specificity, positive and negative predictive values for choledocholithiasis of MRCP and IOC were identified in order to detect if MRCP could replace IOC in prediction of choledocholithiasis. Sensitivity of U/S, MRCP and IOC were $87.5 \%, 77.8 \%$ and $87.5 \%$ respectively. Specificity of these methods revealed $42.8 \%$ for U/S, $65 \%$ for MRCP and $100 \%$ for IOC. These results reveal that IOC still has a higher specificity and sensitivity over MRCP in prediction of CBD stones. Positive predictive values (PPV) of U/S, MRCP and IOC were $36.8 \%, 50 \%$ and $100 \%$ while negative predictive values (NPV) were $90 \%$ for U/S, $86.7 \%$ for MRCP and $95.5 \%$ for IOC. With an incomparable PPV, IOC could be considered a good positive test in detection of choledocholithiasis with higher accuracy than MRCP. It was also noted that all these measures had high NPVs so, they were could be considered good negative tests in ruling out choledocholithiasis.

According to Tofigh et al. experience in 2013, the positive predictive value (PPV) for IOC was $88 \%$ while it was $43 \%$ for MRCP. The diagnostic accuracy of IOC was $98 \%$ and $85 \%$ for MRCP. The results suggested that IOC is much more accurate in prediction of CBD stones. There were no significantly different results in specificity or sensitivity of both methods. From these results, the researchers concluded that MRCP could not omit the need foruse of IOC and supposed that routine MRCP use as a substitution of IOC procedure needs further more investigations on larger numbers of patients ${ }^{(20)}$.

Ward et al. reported that MRCP showed significant effect on the treatment of patients with choledocholithiasis. MRCP did not affect length of hospital stay and contributed to the 95\% PPV. These results illustrated the accuracy of MRCP in cases of suspected choledocholithiasis patients at a low cost with regard to risk and time ${ }^{(21)}$.

In the study of Lin $\mathrm{C}$ et al., revealed that initial $\mathrm{LC}+\mathrm{IOC}$ is associated with a shorter hospital stay compared to LC with preoperative MRCP or ERCP in the management of choledocholithiasis. They recommended LC+ IOC procedure as the optimal treatment choice for suspected choledocholithiasis ${ }^{(22)}$.

However, other studies that compared MRCP to IOC showed that MRCP had a higher sensitivity and specificity $(3,7,23,24)$. Therefore, they concluded that MRCP could replace the IOC for diagnosis of choledocholithiasis based on results indicating that MRCP is a sensitive and specific diagnostic procedure (7). These results did not agree with current results so, more studies on larger samples are needed. Hallal et al. study identified high accuracy of MRCP and concluded that patients with treated gallstone pancreatitis do not need preoperative ERCP or IOC if having a negative MRCP and patients with a positive MRCP should have a preoperative ERCP ${ }^{(25)}$.

Dilated CBD was reported in $65.5 \%$ of cases by $\mathrm{U} / \mathrm{S}$ and in $48.3 \%$ MRCP. This clarifies the diagnostic value of MRCP over U/S in preoperative diagnosis of CBD stones. While abnormal cholangiograms in the study were noticed only in 24.1 $\%$ and all these cases needed further intervention. Also, in the 15 cases with normal MRCP, one case had abnormal cholangiogram. So, IOC had a higher sensitivity, specificity and PPV over other methods including MRCP and treated postoperative ERCP 24 hours after surgery.

During the postoperative follow-up, single case developed obstructive jaundice one month after operation despite normal U/S, MRCP and IOC; and treated by ERCP stone extraction. So, there was only one case $(3 \%)$ of missed CBD stones in the study.

IOC was helpful in detection of 2 cases of CBD stones despite no history of obstructive jaundice. Use of IOC saved those patients from unnecessary preoperative ERCP in 8 patients with positive history of obstructive jaundice with normal IOC. In this experience, history of obstructive jaundice was reported in $44.9 \%$ of the cases reporting low sensitivity $(71.5 \%)$ and specificity $(63.6 \%)$ and also low positive predictive value $(38.5 \%)$ in detection of choledocholithiasis. This indicates a poor predictive value for detection of choledocholithiasis but still have a high negative predictive value (87.5\%) so, absence of obstructive jaundice in history is considered a good negative test. According to these results, it was recommended that further preoperative investigations and IOC should be done to before decision of ERCP on is taken based on history of obstructive jaundice.

\section{CONCLUSION}

Despite the importance of preoperative diagnostic tools, IOC is a very useful procedure that 
provide detailed biliary anatomy and could be done in selected cases for detection of choledocholithiasis in suspected cases, avoidance of unnecessary ERCP in most patients with non-specific CBD dilatation and prevention of biliary injuries in difficult cases with high risk for injury.

\section{REFERENCES}

1. Perissat J, Huibregste $\mathrm{K}$, Keane FB, Russel RC, Neoptolemos JP (1994): Management of bile duct stones in the era of laparoscopic cholecystectomy. Br J Surg., 81:799-810.

2. Heaton KW, Braddon FE, Mountford RA, Hughes AO and Emmett PM (1991): Symptomatic and silent gall stones in the community. Gut, 32:316-20.

3. Shanmugam V, Beattie GC, Yule SR, Reid W, Loudon MA (2005): Is magnetic resonance cholangiopancreatography the new gold standard in biliary imaging? Br J Radiol., 78:888-93.

4. Mercer S, Mr, Singh S, Paterson I (2007): Selective MRCP in the management of suspected common bile duct stones. HPB (Oxford), 9:125-30.

5. Paul A, Millat B, Holthausen U, Sauerland S, Neugebauer E, Berthou JC, Brambs HJ, DominguezMuñoz JE, Goh P, Hammarström LE and Lezoche E (1998): Diagnosis and treatment of common bile duct stones (CBDS). Surgical endoscopy, 12(6):856-864.

6. Soto JA, Barish MA, Yucel EK, Siegenberg D, Ferrucci JT and Chuttani R (1996): Magnetic resonance cholangiography: comparison with endoscopic retrograde cholangiopancreatography. Gastroenterology, 110(2):589597.

7. Dalton SJ, Balupuri $S$ and Guest $\mathbf{J}$ (2005): Routine magnetic resonance cholangiopancreatography and intraoperative cholangiogram in the evaluation of common bile duct stones. Ann R Coll Surg Engl., 87:469-70.

8. Corbitt JD and Yusem SO (1994): Laparoscopic cholecystectomy with operative cholangiogram. Surg Endosc., 8:292-5.

9. Katz D, Nikfarjam M, Sfakiotaki A and Christophi C (2004): Selective endoscopic cholangiography for the detection of common bile duct stones in patients with cholelithiasis. Endoscopy, 36:1045 1049.

10. Collins C, Maguire D, Ireland A, Fitzgerald $E$ and O'Sullivan GC (2004): A prospective study of common bile duct calculi in patients undergoing laparoscopic cholecystectomy: natural history of choledocholithiasis revisited. Ann Surg., 239:28-33.

11. Ghazal AH, Sorour MA, El-Riwini M and El-Bahrawy H (2009): Single-step treatment of gall bladder and bile duct stones: A combined endoscopic-laparoscopic technique. International Journal of Surgery, 7(4): 338-346.

12. Overby DW, Apelgren KN, Richardson W and Fanelli R (2010): Society of American Gastrointestinal and Endoscopic Surgeons. SAGES' guidelines for the clinical application of laparoscopic biliary tract surgery. Surg Endosc., 24:2368-2386.

13. Eikermann M, Siegel R, Broeders I, Dziri C, Fingerhut A and Gutt C (2012): Prevention and treatment of bile duct injuries during laparoscopic cholecystectomy: the clinical practice guidelines of the European Association for Endoscopic Surgery (EAES). Surg Endo., 26:3003-3039.

14. Amott D, Webb A and Tulloh B (2005): Prospective comparison of routine and selective operative cholangiography. ANZ J Surg, 75:378-382.

15. Ludwig K, Bernhardt J, Steffen H, Lorenz D (2002): Contribution of intraoperative cholangiography to incidence and outcome of common bile duct injuries during laparoscopic cholecystectomy. Surg Endosc., 16:10981104.

16. Flum DR, Dellinger EP, Cheadle A, Chan L and Koepsell T (2003): Intraoperative cholangiography and risk of common bile duct injury during cholecystectomy. J Am Med Assoc., 289:1639-1644.

17. Sheffield KM, Riall TS, Han Y, Kuo YF, Townsend Jr CM and Goodwin JS (2013): Association between cholecystectomy with vs without intraoperative cholangiography and risk of common duct injury. J Am Med Assoc., 310:812-820.

18. Ford JA, Soop M, Du J, Loveday BP and Rodgers $M$ (2012): Systematic review of intraoperative cholangiography in cholecystectomy. Br J Surg, 99:160167.

19. Ragulin-Coyne E, Witkowski ER, Chau Z, Chau NS, Santry HP, Callery MP and Tseng J (2013): Is routine intraoperative cholangiogram necessary in the twenty first century? A national view. J Gastrointest surg., 17:434-442.

20. Tofigh AM, Razmjoie F, Khabbaz A, Ayazi K, Farahmand S, Honar BN, and Nikshoar MR (2013): Comparing the efficacy of preoperative magnetic resonance cholangiopancreatography with intra-operative cholangiography in patients suspicious to biliary stones. Gastroenterology and hepatology from bed to bench, 6(2): 80.

21. Ward WH, Fluke LM, Hoagland BD, Zarow GJ, Held JM and Ricca RL (2015): The role of magnetic resonance cholangiopancreatography in the diagnosis of choledocholithiasis: do benefits outweigh the costs? The American surgeon, 81(7): 720-725.

22. Lin C, Collins J N, Britt R C and Britt L D (2015): Initial cholecystectomy with cholangiography decreases length of stay compared to preoperative MRCP or ERCP in the management of choledocholithiasis. The American Surgeon, 81(7): 726-731.

23. Alcaraz M J, Morena E J, Polo A, Ramos A, Cal De M A and González A (2000): A comparative study of magnetic resonance cholangiography and direct cholangiography. Rev Esp Enferm Dig., 92:427-38.

24. Varghese J C, Liddell R P, Farrell M A, Murray F E, Osborne $H$ and Lee M J (2000): Diagnostic accuracy of magnetic resonance cholangiopancreatography and ultrasound compared with direct cholangiography in the detection of choledocholithiasis. Clin. Radiol., 55:25-35.

25. Hallal A H, Amortegui J D, Jeroukhimov I M, Casillas J, Schulman C I, Manning R J and Sleeman D (2005): Magnetic resonance cholangiopancreatography accurately detects common bile duct stones in resolving gallstone pancreatitis. Journal of the American College of Surgeons, 200(6): 869-875. 